\title{
Tratamiento farmacológico de la hiperplasia prostática benigna. Revisión de la bibliografía
}

\section{Pharmacologic treatment of benign prostatic hyperplasia: A literature review}

Hugo López-Ramos,,$^{1,3}$ Mauricio Medina-Rico, ${ }^{2,3}$ Danielle Bastidas, ${ }^{3}$ Brunno Lara ${ }^{4}$

\begin{abstract}
Resumen
La hiperplasia prostática benigna afecta a 50\% de la población mundial, principalmente a pacientes de 70-80 años de edad. Actualmente existen diferentes tratamientos, pero el farmacológico se considera el de primera línea. El objetivo de esta revisión fue encontrar la bibliografía relacionada con el tratamiento farmacológico de la hiperplasia prostática benigna. Para la investigación se revisaron las bases de datos y repositorios: Medline, Embase, Central, Scopus y LILACS. Solo se incluyeron estudios clínicos que evaluaron la eficacia de los diferentes fármacos indicados a pacientes con hiperplasia prostática benigna. Se registraron 1595 referencias revisadas por título y resumen, pero solo se seleccionaron 49 para la revisión de texto completo. Los medicamentos identificados fueron: tamsulosina, alfusosina, doxazosina, silodosina, dutasterida, finasterida, fesoterodina tadalafilo, sildenafilo y naftopidilo. Además, se encontraron estudios de fitoterapia que evaluaron la eficacia de Serenoa repens. La mayor parte de los medicamentos son efectivos para el tratamiento de los síntomas urinarios provocados por la hiperplasia prostática benigna. Los resultados coinciden con las recomendaciones de las últimas guías de práctica clínica. La elección del medicamento de primera línea depende del médico tratante, quien debe considerar, principalmente, los posibles eventos adversos y las preferencias del paciente.
\end{abstract}

PALABRAS CLAVE: Hiperplasia prostática benigna; tratamiento farmacológico; fitoterapia, síntomas de la vía urinaria baja.

\section{Abstract}

Benign prostatic hyperplasia affects $50 \%$ of the population and is more frequent in the seventh and eighth decades of life. There are currently different treatments for benign prostatic hyperplasia and pharmacologic management is considered first-line treatment. The aim of the present study was to conduct a literature review related to the pharmacologic management of benign prostatic hyperplasia, utilizing the following 5 databases: Medline, Embase, Central, Scopus, and LILACS. Only the clinical studies that evaluated the efficacy of the different drugs employed for the symptomatic management of the patient with benign prostatic hyperplasia were included. A total of 1595 references were reviewed by title and abstract, and 49 final references were selected for complete text review. The medications identified were: tamsulosin, alfuzosin, doxazosin, silodosin, dutasteride, finasteride, fesoterodine, tadalafil, sildenafil, and naftopidil. In addition, studies on phytotherapy that evaluated the effectiveness of Serenoa repens were included. The majority of the therapeutic options appear to be effective for the treatment of urinary symptoms caused by benign prostatic hyperplasia and the results identified are in accordance with the recommendations of the latest clinical practice guidelines. The choice of a first-line medication depends on the treating physician who must consider both the possible adverse events and the preferences of the patient.

KEYWORDS: Benign prostatic hyperplasia; Medical management; Pharmacological management; Phytotherapy; Low urinary tract symptoms.

\footnotetext{
${ }^{1}$ Departamento de Urología. Hospital Universitario San Ignacio, Bogotá, Colombia.

2 Departamento de Epidemiología Clínica y Bioestadística, Hospital Universitario San Ignacio, Bogotá, Colombia. ${ }^{3}$ Facultad de Medicina, Pontificia Universidad Javeriana, Bogotá, Colombia. ${ }^{4}$ Facultad de Medicina, Universidad Nacional de Colombia.
}

Recibido: marzo 2018

Aceptado: julio 2018

Correspondencia

Mauricio Medina Rico

medina.mauricio@javeriana.edu.co

Este artículo debe citarse como López-Ramos H, Medina-Rico M, Bastidas D, Lara B. Tratamiento farmacológico de la hiperplasia prostática benigna. Revisión de la bibliografía. Rev Mex Urol. 2018 julio-agosto;78(4):321-334. DOI: https://doi.org/10.24245/revmexurol.v78i4.2093 


\section{ANTECEDENTES}

La hiperplasia prostática benigna es una enfermedad que provoca síntomas urinarios moderados a severos en $50 \%$ de la población de entre 70-80 años de edad. ${ }^{1}$ Los síntomas se originan por la obstrucción de la vía urinaria baja y se clasifican en: obstructivos (micción urinaria débil, esfuerzo abdominal, dificultad para iniciar la micción, micción intermitente, evacuación vesical incompleta y goteo posmiccional) e irritativos (polaquiuria, nicturia, urgencia miccional, incontinencia por urgencia miccional y disuria). ${ }^{2}$ El tamaño de la próstata no siempre se correlaciona con la severidad de los síntomas, sino que existen factores asociados, como las propiedades de la cápsula prostática. ${ }^{3}$

Si bien la evaluación de la hiperplasia prostática benigna se realiza por diferentes métodos y técnicas, como tacto rectal, ecografía o determinación del antígeno prostático, ${ }^{1}$ la manifestación de los síntomas es meramente clínica; por tanto, suelen utilizarse escalas o índices (cuestionarios) para estandarizar su gravedad. En este caso, el IPSS (International Prostate Symptom Score) clasifica los síntomas en: leves (0-7 puntos), moderados (8-19 puntos) o severos (20-35 puntos). ${ }^{2}$

Aunque el tratamiento inicial de la hiperplasia prostática benigna fue durante mucho tiempo la cirugía, ésta ha disminuido radicalmente a través de los años, debido al advenimiento de diferentes alternativas médicas, donde el protocolo farmacológico representa una opción apropiada para pacientes con síntomas leves a moderados. ${ }^{1}$ Entre los fármacos más prescritos se encuentran:

a. Bloqueadores de los receptores $\alpha-1$ : ejercen su función en los receptores $\alpha-1$, especialmente en los tipos A y B; su mecanismo de acción consiste en relajar el músculo liso prostático, facilitar la micción y disminuir la resistencia uretral, sin reducir la contractilidad del detrusor. ${ }^{4}$ b. Inhibidores de la 5-alfa-reductasa (5ARI): la 5- $\alpha$-reductasa tiene la capacidad de almacenar dihidrotestosterona prostática cuando se encuentra en bajas concentraciones. Los 5-ARI disminuyen la proliferación celular mediante la inhibición directa de la conversión de testosterona en el tejido prostático. ${ }^{4}$

c. Anticolinérgicos: se prescriben para el tratamiento de pacientes con urgencia e incontinencia urinaria de urgencia ocasionadas por la hiperactividad vesical secundaria a la obstrucción del conducto urinario de salida. Estos medicamentos se descontinuaron debido a la alta tasa de retención urinaria; sin embargo, pueden indicarse en combinación con otros fármacos. ${ }^{4}$

d. Tratamientos combinados: la combinación más frecuente incluye un bloqueador $\alpha$ y un inhibidor de la 5-alfa-reductasa, incluso un bloqueador alfa y un anticolinérgico. ${ }^{5}$

En la actualidad también se evalúa el uso de la fitoterapia para disminuir los síntomas de los pacientes con hiperplasia prostática benigna. Es probable que su mecanismo de acción se asocie con la inhibición no competitiva de los inhibidores 1 y 2 de la 5 - $\alpha$-reductasa, además de tener participación importante como antiedematoso, antiestrogénico y antiinflamatorio. ${ }^{4}$

Puesto que existen diversos fármacos y esquemas de tratamiento para la hiperplasia prostática benigna, además de la eficacia y abundante información de cada uno, el objetivo de este estudio fue revisar la bibliografía disponible, describir y sintetizar la información relacionada con estos medicamentos y facilitar la toma de decisiones del personal médico en la práctica clínica. 


\section{METOdOLOGÍA}

Revisión bibliográfica, con una aproximación a la estrategia Cochrane de revisiones sistemáticas, relacionada con el tratamiento farmacológico y disminución de los síntomas de la vía urinaria baja provocados por hiperplasia prostática benigna. Para la investigación se revisaron las bases de datos y repositorios: Medline, Embase, Central, Scopus y Lilacs. Se establecieron los criterios de búsqueda en forma de texto libre y términos indizados. Para caracterizar la hiperplasia prostática benigna se utilizaron las palabras clave: "prostatic hyperplasia", y "benign prostatic hyperplasia"; en relación con el tratamiento farmacológico se incluyeron los términos: "drug therapy", "complementary therapies", "medical management", "pharmacological treatment", "medicinal plant", "medicinal herb", "phytotherapy", "alternative treatment", "alternative medicine", "alternative therapy" $y$ "non surgical treatment". Para encontrar posibles artículos de literatura gris se consultó el National Technical Information Service (NTIS) y la Asociación Europea de Literatura Gris (EAGLE), además de la búsqueda en línea con Google académico, con información o artículos publicados en los últimos 5 años.

Criterios de inclusión: ensayos clínicos controlados aleatorizados, con información de la efectividad de los diferentes tratamientos farmacológicos para disminuir los síntomas de la vía urinaria baja en pacientes con hiperplasia prostática benigna, ya sea mediante la comparación de dos o más protocolos diferentes, o con dos o más esquemas del mismo tratamiento. Se seleccionaron estudios con pacientes de entre 40 y 80 años de edad, con síntomas urinarios moderados (mayor de 7), según la clasificación de la escala IPSS.

Criterios de exclusión: estudios que evaluaron la efectividad de los diferentes protocolos médicos en pacientes con hiperplasia prostática benigna y enfermedades concomitantes que alteraron la evolución de los síntomas de la vía urinaria baja, por ejemplo: vejiga hiperactiva o disfunción eréctil, con el propósito de evaluar la disminución de los síntomas de dichas comorbilidades. Igualmente se excluyeron los artículos que no evaluaron objetivamente la disminución de síntomas mediante la Escala Internacional de Síntomas Prostáticos (IPSS).

Se revisaron las referencias bibliográficas por título, resumen y abstract, por dos revisores de forma independiente. A partir de la primera selección de artículos se analizaron las referencias en texto completo, para comprobar que brindaran datos de interés para el estudio. Los artículos duplicados se excluyeron, así como los que tuvieron un idioma diferente al inglés o español. De los estudios incluidos se extrajo la información reportada en sus resultados y se analizaron los métodos y discusiones para encontrar posibles dificultades $y$ fortalezas de cada investigación (Figura 1).

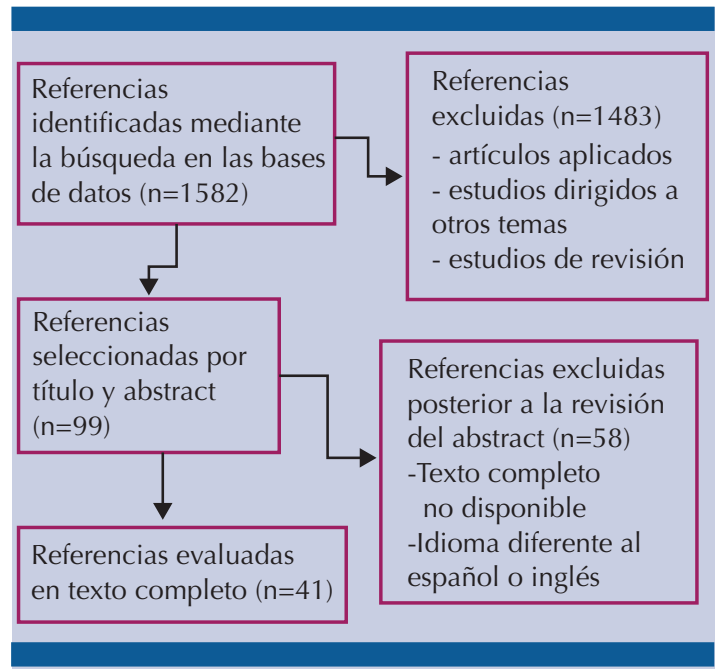

Figura 1. Diagrama de flujo de la búsqueda bibliográfica.

\section{RESULTADOS}

De acuerdo con la búsqueda de la información, se encontraron 1595 referencias revisadas por título, 
resumen y abstract; sin embargo, se seleccionaron 89 artículos para revisión en texto completo, de los que se excluyeron 55 por no especificar la población de estudio, había comorbilidades asociadas o no incluían métodos objetivos para evaluar la disminución de los síntomas. Las referencias sin texto completo (formato de poster o abstract) también fueron excluidas. Finalmente se analizaron 49 artículos en texto completo para la extracción de los datos (Cuadro 1).

Bloqueadores de los receptores $\alpha-1$

\section{Silodosina}

Se encontraron seis estudios efectuados en poblaciones similares: hombres mayores de 50 años, con síntomas de la vía urinaria baja moderados a graves, con IPSS mayor de 8. Novara y su grupo evaluaron la eficacia de silodosina versus placebo y encontraron disminución de los síntomas de 6.6 puntos en el IPSS del grupo de estudio. ${ }^{6}$ Seki y sus colaboradores evaluaron las dosis de silodosina de $4 \mathrm{mg} /$ día vs $4 \mathrm{mg} / 12$ h y observaron que ambas estrategias disminuyeron los síntomas urinarios, según el IPSS, sin encontrar diferencias significativas. ${ }^{7}$ Por su parte, Choo y sus coautores compararon las dosis de silodosina de $8 \mathrm{mg} /$ día versus dos dosis de $4 \mathrm{mg}$ cada 12 horas, sin encontrar diferencias en IPSS o Qmáx. ${ }^{8}$

Dos estudios compararon silodosina con naftopidilo y reportaron mejoría con ambas estrategias; sin embargo, el grupo que recibió silodosina tuvo mejor reacción. ${ }^{9,10}$ En el ensayo de Shirakawa y colaboradores observaron que aunque hubo mejoría con silodosina, no generó ningún cambio en el Qmáx. ${ }^{9}$

Pande y su grupo evaluaron silodosina con tamsulosina y encontraron resultados similares con ambos fármacos en el IPSS. Entre las reacciones adversas reportaron eyaculación retrógrada con silodosina e hipotensión postural con tamsulosina. ${ }^{11}$

\section{Doxazosina}

La investigación de Keten y su equipo de trabajo, en la que evaluaron las dosis de 4 mg/día versus $8 \mathrm{mg} /$ día de doxazosina, reportó eficacia similar con ambos protocolos para el tratamiento los síntomas urinarios de la vía baja; la dosis de $8 \mathrm{mg} /$ día fue una elección eficaz para los pacientes con reacción inadecuada a la dosis de $4 \mathrm{mg} /$ día. $^{12}$

\section{Alfuzosina}

Manjunatha y sus colaboradores analizaron la reacción de alfuzosina versus tamsulosina y silodosina, y encontrando que los tres tratamientos muestran similar efectividad y buena tolerancia. ${ }^{13}$ El estudio de Kumar y su grupo comparó la eficacia de alfuzosina y tadalafil, además del tratamiento combinado entre ambos fármacos, y mostró mejores resultados con alfuzosina y la terapia combinada. ${ }^{14}$

\section{Tamsulosina}

La investigación de Masumori y su grupo, en la que compararon tamsulosina con placebo, reportó la disminución de síntomas urinarios, en promedio, de 5 puntos, según la escala IPSS; no obstante, se consideró que su prescripción a largo plazo podría ser innecesaria, puesto que se observó disminución de síntomas persistentes después de suspender la tamsulosina. ${ }^{15}$ Kim y sus coautores evaluaron la eficacia de tamsulosina, en dosis de 0.2 y $0.4 \mathrm{mg}$, y observaron similar reacción con ambas estrategias, sin cambios estadísticamente significativos. ${ }^{16}$

La comparación entre tamsulosina y silodosina evidenció disminución de los síntomas de la vía urinaria baja, de acuerdo con el índice IPSS, sin diferencia estadísticamente significativa entre ambos fármacos. ${ }^{17} \mathrm{Al}$ evaluar la eficacia de tamsulosina versus tadalafilo se observó, igual- 
mente, similar eficacia; sin embargo, cuando se prescribieron de forma combinada resultó moderadamente mejor. ${ }^{18}$ Oelke y colaboradores reportaron similar efectividad durante 12 semanas de tratamiento con ambos fármacos. ${ }^{19}$

Inhibidores de la 5-fosfodiesterasa

\section{Naftopidil}

Un estudio que comparó naftopidilo versus tamsulosina encontró mejor reacción en pacientes con síntomas obstructivos que recibieron tamsulosina y disminución de los síntomas de urgencia en quienes consumieron naftopidilo. ${ }^{20}$

\section{Vardenafilo}

Gacci y sus colaboradores evaluaron la efectividad de vardenafilo como terapia adicional a la tamsulosina en pacientes con síntomas urinarios provocados por hiperplasia prostática benigna y observaron resultados satisfactorios al prescribir el tratamiento combinado que en monoterapia. ${ }^{21}$

\section{Tadalafilo}

Dmochowski y su grupo estudiaron la efectividad de tadalafilo en dosis de $20 \mathrm{mg} /$ día y encontraron disminución de 5 puntos en la escala de IPSS del grupo intervenido. ${ }^{22}$ Un ensayo que comparó la eficacia de tadalafilo en dosis de $5 \mathrm{mg} /$ día reportó excelentes resultados al disminuir los síntomas urinarios en pacientes sin disfunción eréctil. ${ }^{23}$ Takeda y sus colaboradores evaluaron la dosis de $5 \mathrm{mg}$ de tadalafilo versus placebo, y observaron disminución de 2 puntos en la escala IPSS a partir de la cuarta semana de tratamiento. ${ }^{24}$ Otro estudio realizado por el mismo autor, en el que evaluó la efectividad de tadalafilo a diferentes dosis (2.5 y $5 \mathrm{mg} /$ día) versus placebo, reportó mejor reacción con la dosis de $5 \mathrm{mg} / \mathrm{día}$ en pacientes con síntomas obstructivos. ${ }^{25}$ De manera similar, Yokoyama y sus coautores determinaron la efectividad de tadalafilo en dosis de
2.5 y $5 \mathrm{mg} /$ día versus tamsulosina $0.2 \mathrm{mg}$ /día y encontraron disminución de los síntomas urinarios en los tres grupos; sin embargo, tamsulosina proporcionó mejor reacción en los pacientes. ${ }^{26}$

Singh y sus colaboradores compararon la eficacia de tadalafilo en $10 \mathrm{mg} /$ día, tamsulosina en $0.4 \mathrm{mg} /$ día y la combinación de ambos, y encontraron mejor reacción con esta última estrategia. ${ }^{27}$ Un estudio que evaluó la combinación de tadalafilo de $5 \mathrm{mg}$ /día y tamsulosina de $0.4 \mathrm{mg} /$ día versus tamsulosina en monoterapia, y al igual que el ensayo anterior reportó mejor eficacia el tratamiento combinado en la disminución de los síntomas urinarios por hiperplasia prostática benigna. ${ }^{28}$

Respecto a la evaluación de tadalafilo combinado con finasterida versus finasterida en monoterapia, se observaron mejores resultados con la terapia combinada. ${ }^{29}$

\section{Sildenafilo}

Fawzi y su grupo evaluaron la combinación de Sildenafilo y tamsulosina versus tamsulosina en monoterapia y encontraron mayor efectividad con el tratamiento combinado para disminuir los síntomas de la vía urinaria bajos. ${ }^{30}$

Inhibidores de la 5- $\alpha$-reductasa (5-ARI)

\section{Dutasterida}

Joo y sus coautores siguieron a dos grupos durante un año: el primero recibió $0.2 \mathrm{mg}$ de tamsulosina y el segundo la combinación de 0.2 mg tamsulosina y $0.5 \mathrm{mg}$ de dutasterida. Entre sus resultados observaron mejor reacción con el tratamiento combinado en cuanto a volumen prostático, PSA y Qmáx. ${ }^{31}$ Por su parte, el ensayo de Roehrborn y colaboradores se basó en el estudio COMBAT, con seguimiento en un grupo de pacientes que 
recibieron $0.4 \mathrm{mg}$ de tamsulosina, $0.5 \mathrm{mg}$ de dutasterida y la combinación de ambos; este último mostró mayor eficacia y menor evolución de los síntomas urinarios. ${ }^{32}$

\section{Finasterida}

Odunsaya y su equipo de trabajo compararon la eficacia de finasterida y tamsulosina en monoterapia versus la combinación de ambos durante 6 meses, y encontraron similar reacción en la disminución de síntomas urinarios a 3 meses de iniciar el tratamiento; sin embargo, la terapia combinada mostró resultados sostenidos a 6 meses. ${ }^{33}$

\section{Anticolinérgicos}

\section{Fesoterodina}

Konstantinidis y sus colaboradores efectuaron un estudio en pacientes sin reacción al tratamiento con tamsulosina y los asignaron al azar a dos grupos. El primero continuó con tamsulosina y el segundo recibió tamsulosina y fesoterodina durante 4 semanas. Los resultados mostraron que la terapia combinada benefició a los hombres que no tuvieron mejoría con tamsulosina en monoterapia. $^{34}$

\section{Tolterodina}

Un estudio que comparó la eficacia de tolterodina versus tamsulosina demostró mayor reacción de esta última a largo plazo, con disminución de 3 a 4 puntos en el IPSS a 24 semanas de tratamiento. Así mismo, la terapia combinada reportó reducción entre 8 y 10 puntos de los síntomas urinarios, de acuerdo con el IPSS. ${ }^{35}$

\section{Fitoterapia}

La mayor parte de los artículos se refieren a compuestos como Serenoa repens o Saw palmetto ${ }^{\oplus}$. Ryu y sus coautores compararon la tamsulosina en monoterapia versus la combinación de tamsulosina y Serenoa repens (320 mg/día) y, nuevamente, la terapia combinada ofreció mayores beneficios en cuanto a disminución de los síntomas de almacenamiento urinario. ${ }^{36}$ Por su parte, Argirovic y sus colaboradores observaron tres grupos de pacientes durante seis meses: el primero recibió tamsulosina $0.4 \mathrm{mg} /$ día, el segundo Serenoa repens 320 mg/día y el tercero tamsulosina y Serenoa repens, consiguiendo con esta última beneficios adicionales. ${ }^{37}$

El ensayo de Morgia y su grupo evaluó la efectividad de Profluss ${ }^{\circledast}$ (Serenoa repens, licopeno y selenio) en tres grupos de estudio: el primero recibió Profluss $^{\circledast}$ y placebo, el segundo tamsulosina y placebo, y el tercero tamsulosina y Profluss $^{\circledR}$ durante un año. Entre sus resultados encontraron que la combinación de tamsulosina y Profluss ${ }^{\circledast}$ mostró mayor eficacia en la reducción de los síntomas, según el IPSS, y aumento del Qmáx. ${ }^{38}$

Otro estudio evaluó la eficacia de Longbishu ${ }^{\circledR}$ (tabletas), en el que seleccionaron tres grupos de estudio para recibir Longbishu ${ }^{\circledast}$, doxazosina 4 mg/día o la combinación de doxazosina 4 mg/ día y la cápsula de Longbishu, encontró que cada tratamiento, por sí mismo, es efectivo para el tratamiento de los síntomas urinarios provocados por hiperplasia prostática benigna, comparado con la terapia combinada. ${ }^{39}$ 
López-Ramos $\mathbf{H}$ y col. Hiperplasia prostática benigna

Cuadro 1. Principales resultados de la bibliografía consultada (continúa en la siguiente página)

\begin{tabular}{|c|c|c|c|}
\hline Autor & Población & Intervención & Resultados \\
\hline \multicolumn{4}{|c|}{ Silodosina } \\
\hline $\begin{array}{l}\text { Novara G, } \\
\text { et al. }{ }^{6}\end{array}$ & $\begin{array}{l}847 \text { pacientes, } \\
\text { Edad }>50 \text { años } \\
\quad \text { IPSS } \geq 13 \\
\text { Qmáx } 4 \text { a } 15 \mathrm{~mL} / \mathrm{s}\end{array}$ & $\begin{array}{c}\text { Silodosina } 8 \mathrm{mg} / \text { día por } 12 \text { semanas } \\
\text { vs } \\
\text { placebo }\end{array}$ & $\begin{array}{l}\text { Silodosina disminuyó los síntomas urinarios, } \\
\text { según el IPSS (media de } 6.6 \text { puntos) y Qmáx } \\
\text { de los pacientes. }\end{array}$ \\
\hline $\begin{array}{l}\text { Seki } N \text {, et } \\
\text { al. }{ }^{7}\end{array}$ & $\begin{array}{l}268 \text { pacientes } \\
\text { IPSS } \geq 8 \\
\text { edad }>50 \text { años }\end{array}$ & $\begin{array}{c}\text { Silodosina } 4 \mathrm{mg} / \text { día } \\
\text { vs } \\
\text { Silodosina } 8 \mathrm{mg} / \text { día por } 4 \text { semanas }\end{array}$ & $\begin{array}{l}\text { Silodosina en } 4 \mathrm{mg} / \mathrm{día} \text { y } 4 \mathrm{mg} / 12 \mathrm{~h} \text { disminuye } \\
7 \text { a } 8 \text { puntos del índice IPSS (sin diferencia } \\
\text { estadísticamente significativa). }\end{array}$ \\
\hline $\begin{array}{l}\text { Choo MS, } \\
\text { et al. }{ }^{8}\end{array}$ & $\begin{array}{l}532 \text { pacientes } \\
\text { Edad }>50 \text { años } \\
\quad \text { IPSS } \geq 8 \\
\text { Qmáx }>15 \mathrm{ml} / \mathrm{s}\end{array}$ & $\begin{array}{c}\text { Silodosina } 8 \text { mg/día + placebo } \\
\text { vs } \\
\text { Silodosina } 4 \text { mg/12 horas por } 12 \\
\text { semanas }\end{array}$ & $\begin{array}{l}\text { Ambas estrategias reportaron disminución } \\
\text { cercana a } 7 \text { puntos del IPSS en pacientes con } \\
\text { síntomas de la vía urinaria baja. }\end{array}$ \\
\hline $\begin{array}{l}\text { Shirakawa T, } \\
\text { et al. }{ }^{9}\end{array}$ & $\begin{array}{l}121 \text { pacientes } \\
\text { IPSS } \geq 8, \\
\text { Qmáx }<15 \mathrm{~mL} / \mathrm{s}\end{array}$ & $\begin{array}{c}\text { Silodosina } 8 \text { mg/día } \\
\text { vs } \\
\text { Naftopidilo } 50 \text { mg/día por } 4 \text { u } 8 \\
\text { semanas }\end{array}$ & $\begin{array}{l}\text { Silodosina disminuyó, incluso } 7 \text { puntos del } \\
\text { IPSS, los síntomas urinarios después de } 8 \text { se- } \\
\text { manas de tratamiento. Naftopidilo disminuyó } \\
3 \text { puntos durante el mismo periodo. }\end{array}$ \\
\hline $\begin{array}{l}\text { Yamaguchi } \\
\text { S, et al. }{ }^{10}\end{array}$ & $\begin{array}{l}96 \text { pacientes } \\
\quad \text { IPSS } \geq 8 \\
\text { edad }>50 \text { años }\end{array}$ & $\begin{array}{c}\text { Silodosina } 8 \mathrm{mg} / \text { día } \\
\text { vs } \\
\text { Naftopidilo } 75 \mathrm{mg} / \text { día por } 12 \text { semanas }\end{array}$ & $\begin{array}{l}\text { Silodosina disminuyó } 7 \text { puntos del IPSS los sín- } \\
\text { tomas urinarios a las } 8 \text { semanas de tratamiento } \\
\text { y naftopidilo } 6 \text { puntos en el mismo tiempo. }\end{array}$ \\
\hline \multirow[t]{2}{*}{$\begin{array}{l}\text { Pande S, et } \\
\text { al. }{ }^{11}\end{array}$} & $\begin{array}{l}53 \text { pacientes } \\
\text { Edad }>50 \text { años } \\
\quad \text { IPSS } \geq 8\end{array}$ & $\begin{array}{c}\text { Silodosina } 8 \mathrm{mg} / \text { día } \\
\text { vs } \\
\text { Tamsulosina } 0.4 \mathrm{mg} / \text { día por } 12 \\
\text { semanas }\end{array}$ & $\begin{array}{l}\text { Silodosina y tamsulosina disminuyeron } 11 \text { pun- } \\
\text { tos del IPSS los síntomas urinarios; sin embargo, } \\
\text { no se observaron diferencias significativa con } \\
\text { ambos fármacos. }\end{array}$ \\
\hline & & Doxazosina & \\
\hline $\begin{array}{l}\text { Keten T, et } \\
\text { al. }{ }^{12}\end{array}$ & $\begin{array}{l}162 \text { pacientes } \\
\text { Edad }>45 \text { años } \\
\quad \text { IPSS } \geq 8 \\
\text { Qmáx }>15 \mathrm{~mL} / \mathrm{s}\end{array}$ & $\begin{array}{l}4 \text { mg doxazosina } \\
\text { vs } \\
8 \mathrm{mg} \text { doxazosina }\end{array}$ & $\begin{array}{l}\text { Doxazosina en } 4 \text { y } 8 \text { mg disminuyeron en } 4 \text { y } \\
5 \text { puntos el IPSS, respectivamente, al mes de } \\
\text { tratamiento, con diferencia estadísticamente } \\
\text { significativa. }\end{array}$ \\
\hline \multicolumn{4}{|c|}{ Alfuzosina } \\
\hline $\begin{array}{l}\text { Manjunatha } \\
\mathrm{R} \text {, et al. }{ }^{13}\end{array}$ & $\begin{array}{l}115 \text { pacientes } \\
\text { Edad }>45 \text { años } \\
\quad \text { IPSS } \geq 8\end{array}$ & $\begin{array}{c}\text { Alfuzosina } 10 \mathrm{mg} \\
v s \\
\text { Tamsulosina } 0.4 \mathrm{mg} \\
\text { vs } \\
\text { Silodosina } 8 \mathrm{mg} \text { por } 12 \text { semanas }\end{array}$ & $\begin{array}{l}\text { Alfuzosina, tamsulosina y silodosina mostraron } \\
\text { eficacia similar. }\end{array}$ \\
\hline $\begin{array}{l}\text { Kumar S, et } \\
\text { al. }{ }^{14}\end{array}$ & $\begin{array}{l}75 \text { pacientes } \\
\text { Edad }>50 \text { años } \\
\quad \text { IPSS } \geq 8\end{array}$ & $\begin{array}{c}\text { Alfuzosina } 10 \mathrm{mg} / \text { día } \\
\text { vs } \\
\text { Tadalafilo } 10 \mathrm{mg} / \text { día } \\
\text { vs } \\
\text { Terapia combinada por } 3 \text { meses }\end{array}$ & $\begin{array}{l}\text { Ambos son efectivos en la disminución de los } \\
\text { síntomas de la vía urinaria baja; sin embargo, } \\
\text { alfuzosina es aparentemente mejor. La terapia } \\
\text { combinada proporcionó mejores resultados que } \\
\text { la monoterapia, con disminución media de } 12 \\
\text { puntos del IPSS a las } 12 \text { semanas de tratamiento. }\end{array}$ \\
\hline & & Tamsulosina & \\
\hline
\end{tabular}


Cuadro 1. Principales resultados de la bibliografía consultada (continúa en la siguiente página)

\begin{tabular}{|c|c|c|c|}
\hline Autor & Población & Intervención & Resultados \\
\hline $\begin{array}{l}\text { Masumori } \\
\mathrm{N} \text {, et al. }{ }^{.15}\end{array}$ & $\begin{array}{l}112 \text { pacientes } \\
\text { Edad }>50 \text { años } \\
\quad \text { IPSS } \geq 8\end{array}$ & Tamsulosina 0.4 mg/día por 5 años & $\begin{array}{l}\text { Tamsulosina es eficaz en la reducción de los } \\
\text { síntomas de la vía urinaria baja (disminución } \\
\text { media de } 5 \text { puntos del IPSS). }\end{array}$ \\
\hline $\begin{array}{l}\text { Kim JJ, et } \\
\text { al. }^{16}\end{array}$ & $\begin{array}{l}220 \text { pacientes } \\
\text { Edad }>50 \text { años } \\
\quad \text { IPSS } \geq 12 \\
\text { Qmáx }<15 \mathrm{~mL} / \mathrm{s}\end{array}$ & $\begin{array}{l}\text { Tamsulosina } 0.2 \mathrm{mg} / \text { día } \\
\text { Tamsulosina } 0.4 \mathrm{mg} / \text { día } \\
\text { por } 12 \text { semanas }\end{array}$ & $\begin{array}{l}\text { Ambos fármacos mostraron similar eficacia } \\
\text { (reducción promedio de } 3 \text { a } 4 \text { puntos del IPSS). }\end{array}$ \\
\hline $\begin{array}{l}\text { Takeshita H, } \\
\text { et al. }{ }^{17}\end{array}$ & $\begin{array}{l}34 \text { pacientes } \\
\text { Edad }>50 \text { años } \\
\quad I P S S \geq 8\end{array}$ & $\begin{array}{c}\text { Grupo1: } 4 \text { semanas de silodosina } 4 \\
\text { mg/día, seguido de tamsulosina } 0.2 \\
\text { mg/día por } 4 \text { semanas } \\
\text { Grupo 2: tamsulosina } 0.2 \text { mg/día por } \\
4 \text { semanas, seguido de silodosina } 4 \\
\text { mg/día por } 4 \text { semanas }\end{array}$ & $\begin{array}{l}\text { Silodosina y tamsulosina tuvieron similar efica- } \\
\text { cia, con reducción de hasta } 6 \text { puntos del IPSS y } \\
\text { de los síntomas de la vía urinaria baja. }\end{array}$ \\
\hline $\begin{array}{l}\text { Karami H, } \\
\text { et al. }{ }^{18}\end{array}$ & $\begin{array}{l}183 \text { pacientes } \\
\text { Edad }>45 \text { años } \\
\quad \text { IPSS } \geq 12\end{array}$ & $\begin{array}{l}\text { Grupo 1: tamsulosina } 0.4 \mathrm{mg} / \text { día } \\
\text { Grupo 2: tadalafilo } 20 \mathrm{mg} \text { /día } \\
\text { Grupo 3: tamsulosina + tadalafilo }\end{array}$ & $\begin{array}{l}\text { Ambos medicamentos en monoterapia disminu- } \\
\text { yeron los síntomas urinarios en } 7 \text { puntos según } \\
\text { el IPSS; sin embargo, la terapia combinada fue } \\
\text { relativamente mejor. }\end{array}$ \\
\hline \multirow[t]{2}{*}{$\begin{array}{l}\text { Oelke } M \text {, et } \\
\text { al. }{ }^{19}\end{array}$} & $\begin{array}{c}511 \text { pacientes } \\
\text { Edad }>45 \text { años } \\
\quad \text { IPSS } \geq 12 \\
\text { Qmáx } 4 \text { a } 15 \mathrm{~mL} / \mathrm{s}\end{array}$ & $\begin{array}{l}\text { Grupo 1: tamsulosina } 0.4 \mathrm{mg} / \text { día } \\
\text { Grupo 2: tadalafilo } 20 \mathrm{mg} / \text { día } \\
\text { Grupo 3: placebo }\end{array}$ & $\begin{array}{l}\text { Ambos fármacos mostraron similar efectividad } \\
\text { versus placebo en la reducción de los síntomas } \\
\text { urinarios a las } 12 \text { semanas de tratamiento. }\end{array}$ \\
\hline & & \multicolumn{2}{|l|}{ Naftopidilo } \\
\hline \multirow[t]{2}{*}{$\begin{array}{l}\text { Perumal C, } \\
\text { et al. } .^{20}\end{array}$} & $\begin{array}{l}60 \text { pacientes } \\
\text { Edad } 51-70 \text { años, } \\
\quad \text { IPSS } \geq 12\end{array}$ & $\begin{array}{l}\text { Naftopidilo } 50 \text { mg/día vs tamsulosina } \\
0.4 \mathrm{mg} / \text { día por } 4 \text { semanas }\end{array}$ & $\begin{array}{l}\text { Aunque ambos medicamentos disminuyeron en } \\
14 \text { puntos el IPSS, se observó mejor reacción } \\
\text { en el grupo de naftopidilo. }\end{array}$ \\
\hline & & Valdenafilo & \\
\hline \multirow[t]{2}{*}{$\begin{array}{l}\text { Gacci M, et } \\
\text { al. }{ }^{21}\end{array}$} & $\begin{array}{l}60 \text { pacientes } \\
\text { Edad }>40 \text { años } \\
\quad \text { IPSS } \geq 12\end{array}$ & $\begin{array}{c}\text { Grupo 1: tamsulosina } 0.4 \mathrm{mg} / \text { día } \\
\text { Grupo 2: tamsulosina } 0.4 \mathrm{mg} / \mathrm{día}+ \\
\text { vardenafilo } 10 \mathrm{mg} / \text { día por } 12 \mathrm{semanas}\end{array}$ & $\begin{array}{l}\text { La combinación de vardenafilo y tamsulosina } \\
\text { fue más efectiva que la monoterapia. Disminuyó } \\
3 \text { a } 4 \text { puntos el IPSS después de } 12 \text { semanas de } \\
\text { tratamiento. }\end{array}$ \\
\hline & & Tadalafilo & \\
\hline $\begin{array}{l}\text { Dmochows- } \\
\text { ki R, et al. }{ }^{22}\end{array}$ & $\begin{array}{l}200 \text { pacientes } \\
\text { Edad } \geq 40 \text { años } \\
\quad \text { IPSS } \geq 13\end{array}$ & $\begin{array}{c}\text { Tadalafilo } 20 \mathrm{mg} \\
\text { vs } \\
\text { Placebo por } 12 \text { semanas }\end{array}$ & $\begin{array}{l}\text { Tadalafilo disminuyó los síntomas y el IPSS } 5 \\
\text { puntos. }\end{array}$ \\
\hline $\begin{array}{l}\text { Brock G, et } \\
\text { al. }{ }^{23}\end{array}$ & $\begin{array}{c}202 \text { pacientes } \\
\text { Edad } \geq 45 \text { años } \\
\text { IPSS } \geq 13 \\
\text { Qmáx de } 4 \text { a } 25 \\
\mathrm{~mL} / \mathrm{s}\end{array}$ & $\begin{array}{c}\text { Tadalafilo } 5 \mathrm{mg} \\
\text { vs } \\
\text { Placebo por } 4 \text { semanas }\end{array}$ & $\begin{array}{l}\text { Tadalafilo disminuyó } 2 \text { puntos el IPSS y los } \\
\text { síntomas urinarios. }\end{array}$ \\
\hline $\begin{array}{l}\text { Takeda M, } \\
\text { et al. }{ }^{24}\end{array}$ & $\begin{array}{c}610 \text { pacientes } \\
\text { Edad } \geq 45 \text { años } \\
\text { IPSS } \geq 13 \\
\text { Qmáx de } 4 \text { a } 25 \\
\mathrm{~mL} / \mathrm{s}\end{array}$ & $\begin{array}{c}\text { Tadalafilo } 5 \mathrm{mg} / \text { día } \\
\text { vs } \\
\text { Placebo por } 12 \text { semanas }\end{array}$ & $\begin{array}{l}\text { Tadalafilo de } 5 \text { mg mostró eficacia en la re- } \\
\text { ducción de síntomas urinarios desde la cuarta } \\
\text { semana de tratamiento, con disminución de } 2 \\
\text { puntos según el IPSS. }\end{array}$ \\
\hline
\end{tabular}


López-Ramos $\mathbf{H}$ y col. Hiperplasia prostática benigna

Cuadro 1. Principales resultados de la bibliografía consultada (continúa en la siguiente página)

\begin{tabular}{|c|c|c|c|}
\hline Autor & Población & Intervención & Resultados \\
\hline $\begin{array}{l}\text { Takeda M, } \\
\text { et al. }{ }^{25}\end{array}$ & $\begin{array}{c}442 \text { pacientes } \\
\text { Edad } \geq 45 \text { años } \\
\quad \text { IPSS } \geq 13 \\
\text { Qmáx de } 4 \text { a } 15 \\
\mathrm{~mL} / \mathrm{s}\end{array}$ & $\begin{array}{c}\text { Tadalafilo } 2.5 \mathrm{mg} / \text { día } \\
\text { vs } \\
\text { Placebo } \\
v s \\
\text { Tadalafilo } 5 \mathrm{mg} / \text { día por } 42 \text { semanas }\end{array}$ & $\begin{array}{l}\text { Tadalafilo de } 5 \text { mg fue más efectivo para reducir } \\
\text { los síntomas obstructivos de la vía urinaria baja, } \\
\text { con disminución de } 3 \text { puntos del IPSS. }\end{array}$ \\
\hline $\begin{array}{l}\text { Yokoyama } \\
\text { O, et al. }{ }^{26}\end{array}$ & $\begin{array}{c}612 \text { pacientes } \\
\text { Edad } \geq 45 \text { años } \\
\text { IPSS } \geq 13 \\
\text { Qmáx de } 4 \text { a } 15 \\
\mathrm{~mL} / \mathrm{s}\end{array}$ & $\begin{array}{c}\text { Tadalafilo } 5 \mathrm{mg} / \text { día } \\
\text { vs } \\
\text { Tadalafilo } 2.5 \mathrm{mg} / \text { día } \\
\text { vs } \\
\text { Tamsulosina } 0.2 \mathrm{mg} / \text { día por } 12 \\
\text { semanas }\end{array}$ & $\begin{array}{l}\text { Las tres estrategias disminuyeron los síntomas } \\
\text { urinarios, en aproximadamente } 5 \text { puntos del } \\
\text { IPSS; sin embargo, tamsulosina de } 5 \mathrm{mg} \text { fue } \\
\text { ligeramente mejor. }\end{array}$ \\
\hline $\begin{array}{l}\text { Singh DV, et } \\
\text { al. }{ }^{27}\end{array}$ & $\begin{array}{l}133 \text { pacientes } \\
\text { Edad } \geq 45 \text { años } \\
\quad \text { IPSS }>8\end{array}$ & $\begin{array}{c}\text { Tamsulosina } 0.4 \mathrm{mg} / \text { día } \\
\text { vs } \\
\text { Tadalafilo } 10 \mathrm{mg} / \text { día } \\
\text { vs } \\
\text { Tamsulosina } 0.4 \mathrm{mg} / \text { día + tadalafilo } \\
10 \mathrm{mg} / \text { día por } 12 \text { semanas. }\end{array}$ & $\begin{array}{l}\text { Aunque tamsulosina y tadalafilo fueron efec- } \\
\text { tivos para disminuir los síntomas de la vía } \\
\text { urinaria baja, con reducción de } 10 \text { y } 7 \text { puntos } \\
\text { del IPSS, respectivamente, la terapia combinada } \\
\text { fue evidentemente mejor. }\end{array}$ \\
\hline $\begin{array}{l}\text { Regadas R, } \\
\text { et al. }{ }^{28}\end{array}$ & $\begin{array}{l}40 \text { pacientes } \\
\text { Edad } \geq 45 \text { años } \\
\quad I P S S>14\end{array}$ & $\begin{array}{c}\text { Tadalafilo } 5 \text { mg/día + tamsulosina } 0.4 \\
\text { mg/día } \\
\text { vs } \\
\text { Tamsulosina } 0.4 \text { mg/día + placebo por } \\
4 \text { semanas }\end{array}$ & $\begin{array}{l}\text { La terapia combinada con tadalafilo fue más } \\
\text { efectiva que la monoterapia de tamsulosina, } \\
\text { con reducción de } 3.7 \text { puntos del IPSS, lo que } \\
\text { representó una diferencia estadísticamente } \\
\text { significativa. }\end{array}$ \\
\hline \multirow[t]{2}{*}{$\begin{array}{l}\text { Casabe A, } \\
\text { et al. }{ }^{29}\end{array}$} & $\begin{array}{c}696 \text { pacientes } \\
\text { Edad } \geq 45 \text { años } \\
\quad \text { IPSS } \geq 13 \\
\text { Qmáx de } 4 \text { a } 15 \\
\mathrm{~mL} / \mathrm{s}\end{array}$ & $\begin{array}{c}\text { Tadalafilo }+ \text { finasterida } \\
\text { vs } \\
\text { Placebo + finasterida por } 26 \text { semanas }\end{array}$ & $\begin{array}{l}\text { La combinación de tadalafilo y finasteride } \\
\text { proporcionó mejor reacción que la } \\
\text { monoterapia con finasterida. }\end{array}$ \\
\hline & & Sildenafilo & \\
\hline $\begin{array}{l}\text { Fawzi A, et } \\
\text { al. }{ }^{30}\end{array}$ & $\begin{array}{l}150 \text { pacientes } \\
\text { Edad } \geq 50 \text { años } \\
\quad \text { IPSS } \geq 13\end{array}$ & $\begin{array}{c}\text { Tamsulosina } 0.4 \mathrm{mg}+\text { sildenafilo } 25 \\
\mathrm{mg} \\
\text { vs } \\
\text { Tamsulosina } 0.4 \mathrm{mg}+\text { placebo }\end{array}$ & $\begin{array}{l}\text { La combinación de ambos fármacos disminuyó } \\
3 \text { puntos más, según el IPSS, los síntomas uri- } \\
\text { narios que la monoterapia. }\end{array}$ \\
\hline \multicolumn{4}{|c|}{ Dutasterida } \\
\hline $\begin{array}{l}\text { Joo KL, et } \\
\text { al. }{ }^{31}\end{array}$ & $\begin{array}{c}216 \text { pacientes } \\
\text { Edad }>40 \text { años } \\
\quad \text { IPSS } \geq 13 \\
\text { Qmáx de } 4 \text { a } 15 \\
\mathrm{~mL} / \mathrm{s}\end{array}$ & $\begin{array}{c}\text { Tamsulosina } 0.2 \mathrm{mg} / \text { día } \\
\text { vs } \\
\text { Tamsulosina } 0.2 \mathrm{mg} / \text { día + dutasterida } \\
0.5 \mathrm{mg} / \text { día por } 12 \text { meses }\end{array}$ & $\begin{array}{l}\text { Ambas estrategias se consideraron el mejor } \\
\text { tratamiento en pacientes con síntomas de la } \\
\text { vía urinaria baja, con disminución de } 6.7 \text { y } 7.4 \\
\text { puntos del IPSS en cada grupo. Esta diferencia } \\
\text { es ligeramente mayor conforme aumenta el } \\
\text { volumen prostático. }\end{array}$ \\
\hline $\begin{array}{l}\text { Roehrborn } \\
\text { C, et al. }{ }^{32}\end{array}$ & $\begin{array}{l}3195 \text { pacientes } \\
\text { Edad } \geq 50 \text { años } \\
\quad \text { IPSS } \geq 12, \\
\text { Qmáx } 4-15 \mathrm{~mL} / \mathrm{s}\end{array}$ & $\begin{array}{c}\text { Dutasterida } 0.5 \mathrm{mg} / \text { día + tamsulosina } \\
0.4 \mathrm{mg} / \text { día } \\
\text { vs } \\
\text { Dutasterida } 0.5 \mathrm{mg} / \text { día } \\
\text { vs } \\
\text { Tamsulosina } 0.4 \mathrm{mg} / \text { día }\end{array}$ & $\begin{array}{l}\text { Ambas terapias son efectivas en la reducción de } \\
\text { los síntomas urinarios; sin embargo, la terapia } \\
\text { combinada proporcionó mejores beneficios. }\end{array}$ \\
\hline
\end{tabular}


Cuadro 1. Principales resultados de la bibliografía consultada (continuación)

\begin{tabular}{|c|c|c|c|}
\hline Autor & Población & Intervención & Resultados \\
\hline \multicolumn{4}{|c|}{ Finasterida } \\
\hline \multirow[t]{2}{*}{$\begin{array}{l}\text { Odusanya } \\
\text { B, et al. }{ }^{33}\end{array}$} & $\begin{array}{l}592 \text { pacientes } \\
\text { Edad } \geq 40 \text { años } \\
\text { IPSS } \geq 13\end{array}$ & $\begin{array}{c}\text { Tamsulosina } 0.4 \mathrm{mg} / \text { día } \\
\text { vs } \\
\text { Finasterida } 5 \mathrm{mg} \\
\text { vs } \\
\text { Tamsulosina } 0.4 \mathrm{mg} / \text { día }+ \text { finasterida } 5 \\
\text { mg por } 6 \text { meses }\end{array}$ & $\begin{array}{l}\text { Aunque las monoterapias mostraron eficacia } \\
\text { similar en la reducción de los síntomas urina- } \\
\text { rios ( } 7 \text { puntos del IPSS a } 3 \text { meses), la terapia } \\
\text { combinada generó los mejores resultados a } \\
6 \text { meses de tratamiento, con reducción de } 10 \\
\text { puntos del IPSS. }\end{array}$ \\
\hline & & Fesoterodina & \\
\hline \multirow[t]{2}{*}{$\begin{array}{l}\text { Konstanti- } \\
\text { nidis C, et } \\
\text { al. }^{34}\end{array}$} & $\begin{array}{l}173 \text { pacientes } \\
\text { Edad } \geq 50 \text { años } \\
\quad \text { IPSS } \geq 13\end{array}$ & $\begin{array}{c}\text { Tamsulosina } 0.4 \mathrm{mg} / \text { día } \\
\text { vs } \\
\text { Tamsulosina } 0.4 \mathrm{mg} / \text { día + fesoterodina }\end{array}$ & $\begin{array}{l}\text { La combinación de ambos fármacos fue signi- } \\
\text { ficativamente más efectiva que la monoterapia. }\end{array}$ \\
\hline & & Tolterodina & \\
\hline \multirow[t]{2}{*}{$\begin{array}{l}\text { Cai JL } L_{\llcorner} \text {et } \\
\text { al. }{ }^{35}\end{array}$} & $\begin{array}{l}152 \text { pacientes } \\
\text { Edad } \geq 50 \text { años } \\
\quad \text { IPSS } \geq 13\end{array}$ & $\begin{array}{c}\text { Tamsulosina } 0.2 \mathrm{mg} / \text { día } \\
\text { vs } \\
\text { Tolterodina } 4 \mathrm{mg} \\
\text { vs } \\
\text { Tamsulosina + tolterodina por } 24 \\
\text { semanas }\end{array}$ & $\begin{array}{l}\text { Los medicamentos en monoterapia disminu- } \\
\text { yeron los síntomas urinarios a } 12 \text { meses de } \\
\text { tratamiento. Tamsulosina fue más efectiva que } \\
\text { tolterodina a largo plazo; no obstante, la terapia } \\
\text { combinada fue proporcionó mejores resultados. }\end{array}$ \\
\hline & & Fitoterapia & \\
\hline $\begin{array}{l}\text { Ryu WY, et } \\
\text { al. }{ }^{36}\end{array}$ & $\begin{array}{l}120 \text { Pacientes } \\
\text { Edad } 50-80 \text { años } \\
\text { IPSS }>10 \\
\text { Qmáx de } 5 \text { a } 15 \\
\mathrm{~mL} / \mathrm{s}\end{array}$ & $\begin{array}{c}\text { Tamsulosina } 0.2 \mathrm{mg} \text { día } \\
\text { vs } \\
\text { Tamsulosina } 0.2 \mathrm{mg} / \text { día }+ \text { Serenoa } \\
\left.\text { repens (Saw Palmetto }{ }^{\circledR}\right) 320 \mathrm{mg} / \text { día } \\
\text { por } 12 \text { meses }\end{array}$ & $\begin{array}{l}\text { Ambas estrategias disminuyeron entre } 4 \text { y } 6 \\
\text { puntos el IPSS a } 6 \text { y } 12 \text { meses de tratamiento. } \\
\text { El IPSS total no mostró diferencias significativas; } \\
\text { sin embargo, al evaluar la terapia combinada } \\
\text { se observaron diferencias en cuanto a síntomas } \\
\text { de almacenamiento respecta. }\end{array}$ \\
\hline $\begin{array}{l}\text { Argirovic A, } \\
\text { et al. }{ }^{37}\end{array}$ & $\begin{array}{l}297 \text { Pacientes } \\
\text { Edad }>50 \text { años } \\
\quad \text { IPSS }>7 \\
\text { Qmáx de } 5 \text { a } 15 \\
\text { mL/s }\end{array}$ & $\begin{array}{c}\text { Tamsulosina } 0.4 \mathrm{mg} / \text { día } \\
\text { vs } \\
\text { Serenoa repens } 320 \mathrm{mg} / \text { día } \\
\text { vs } \\
\text { Tamsulosina } 320 \mathrm{mg} / \text { día + tamsulosi- } \\
\text { na } 0.4 \mathrm{mg} / \text { día por } 6 \text { meses }\end{array}$ & $\begin{array}{l}\text { Al parecer, Serenoa repens no genera ningún } \\
\text { beneficio adicional en pacientes con síntomas } \\
\text { urinarios provocados por hiperplasia prostática } \\
\text { benigna. }\end{array}$ \\
\hline $\begin{array}{l}\text { Morgia G, } \\
\text { et al. }{ }^{38}\end{array}$ & $\begin{array}{l}225 \text { pacientes } \\
\text { Edad }>55 \text { años } \\
\quad \text { IPSS }>12 \\
\text { Qmáx }<15 \mathrm{~mL} / \mathrm{s}\end{array}$ & $\begin{array}{c}\text { Profluss }^{\circledR}+\text { placebo por } 1 \text { año } \\
\text { vs } \\
\text { Tamsulosina } 0.4 \text { mg/día + placebo por } \\
1 \text { año } \\
\text { vs } \\
\text { Profluss }^{\circledR}+\text { tamsulosina } 0.4 \mathrm{mg} / \text { día } \\
\text { por } 1 \text { año }\end{array}$ & $\begin{array}{l}\text { Profluss }^{\circledR} \text { es eficaz, por sí mismo, en el trata- } \\
\text { miento de pacientes con síntomas de la vía } \\
\text { urinaria baja (reducción de } 3 \text { puntos del IPSS). } \\
\text { A su vez, la terapia combinada es más efectiva } \\
\text { que las terapias individuales para disminuir la } \\
\text { puntuación del IPSS. }\end{array}$ \\
\hline $\begin{array}{l}\text { Song C, et } \\
\text { al. }^{39}\end{array}$ & $\begin{array}{l}360 \text { pacientes } \\
\text { Edad }>50 \text { años } \\
\quad \text { IPSS }>8\end{array}$ & $\begin{array}{c}\text { Longbishu }^{\circledast} \\
\text { vs } \\
\text { Doxazosina } 4 \mathrm{mg} \\
\text { vs } \\
\text { Longbishu }^{\circledR}+\text { doxazosina } 4 \mathrm{mg}^{\text {doxas }}\end{array}$ & $\begin{array}{l}\text { Longbishu }^{\circledR} \text { en monoterpia o combinado con } \\
\text { doxazosina disminuyó significativamente el } \\
\text { puntaje del IPSS en pacientes con hiperplasia } \\
\text { prostática benigna. }\end{array}$ \\
\hline
\end{tabular}




\section{DISCUSIÓN}

En términos generales, todos los tratamientos fueron eficaces para disminuir los síntomas urinarios de pacientes con hiperplasia prostática benigna; sin embargo, los bloqueadores de los receptores $\alpha-1$ son los fármacos con mayor efectividad, seguidos de los inhibidores de la 5-a-reductasa, anticolinérgicos e inhibidores de la 5-fosfodiesterasa. De la misma manera, los estudios que evaluaron la terapia combinada reportaron mayor eficacia que las monoterapias. Aunque algunos estudios encontraron diferencias estadísticas entre una y otra estrategia, rara vez logró ser mayor de 3 o 4 puntos según la escala IPSS; por tanto, es necesario determinar si la diferencia reportada fue clínicamente relevante..$^{40}$ En cuanto a la fitoterapia, se encontraron diferentes opiniones relacionadas con la efectividad de Serenoa repens en monoterapia o en combinación, por lo que su recomendación no es definitiva.

De acuerdo con diversos estudios, los bloqueadores de los receptores $\alpha-1$ se consideran el tratamiento de primera línea en pacientes con síntomas de la vía urinaria baja moderados, provocados por hiperplasia prostática benigna. Incluso los inhibidores de la 5 - $\alpha$-reductasa representan una alternativa eficaz y segura. ${ }^{41}$ En relación con la terapia combinada, se han encontrado similitudes respecto de sus beneficios en pacientes en quienes falla la monoterapia. ${ }^{42}$

Al revisar guías de práctica clínica para el tratamiento farmacológico de la hiperplasia prostática benigna de los grupos NICE (National Institute for Health and Care Excellence), GIN (Guidelines international network), National Guideline Clearinghouse, New Zealand Guidelines, grupo Scielo y el Cenetec (México), se identificaron seis documentos publicados en los últimos 5 años que cumplieron con las recomendaciones del nivel de evidencia. Todas las guías coincidieron en que cada protocolo de tratamiento (bloqueadores de los receptores $\alpha-1$, inhibidores de la 5-alfa-reductasa y anticolinérgicos) es eficaz y seguro en pacientes con síntomas de la vía urinaria baja provocados por hiperplasia prostática benigna, y se les considera dentro de la recomendación grado A, con nivel de evidencia fuerte..$^{43-48}$ Sin embargo, ninguna de las guías de práctica clínica propone una terapia de primera línea definitiva; por el contrario, la indicación o contraindicación de algún medicamento depende de la preferencia del paciente y posibles eventos adversos. Por ejemplo, los bloqueadores de los receptores $\alpha-1$ pueden provocar hipotensión y aumentar el riesgo de recidiva, por lo que deben indicarse con cautela en pacientes de edad avanzada. Así mismo, los inhibidores de la 5-alfa-reductasa disminuyen la libido y la función eréctil; por tanto, deben contraindicarse en adultos jóvenes. ${ }^{45}$ Para facilitar la toma de decisiones médicas es importante considerar elementos adicionales, como el costo-efectividad de cada medicamento. ${ }^{49}$

Las limitaciones de este estudio incluyen su naturaleza de revisión narrativa, con el propósito de actualización de la bibliografía, más allá de ser una revisión sistemática que pretende determinar qué tipo de intervención es mejor; no podemos saber si existe heterogeneidad de los datos expuestos, lo que impide comparar los resultados estadísticamente. Además, nuestros resultados muestran similitud con las últimas guías de práctica clínica y otorgan continuidad de la forma en que se ha atendido al paciente con hiperplasia prostática benigna. Esto resulta importante, pues al realizar la búsqueda de las últimas guías de práctica clínica no se identificó ninguna actualización desde 2015 en América Latina, la última guía en México fue en 2009 y en Colombia en 2014. Este estudio contribuye con la formación del médico general y del especialista, otorgando información suficiente para seguir implementando dichos protocolos en la atención de los pacientes.

En cuanto a la fitoterapia, algunas guías, como la mexicana, la consideran dentro de sus reco- 
mendaciones IIB. ${ }^{48}$ No obstante, debido a las opiniones divergentes acerca de su eficacia, debe continuar la evaluación para posible prescripción en estos pacientes.

\section{CONCLUSIÓN}

Existen múltiples opciones farmacológicas en el tratamiento de pacientes con síntomas de la vía urinaria baja provocados hiperplasia prostática benigna. Dichas estrategias muestran diferencias aparentes en cuanto a eficacia; sin embargo, hasta la fecha no se ha establecido el tratamiento de primera línea. La terapia combinada se describe como segunda línea de tratamiento en pacientes con resistencia a la monoterapia. Para elegir el tratamiento adecuado, el médico debe considerar las diferentes opciones, evaluar los eventos adversos y considerar las preferencias del paciente.

\section{REFERENCIAS}

1. McVary KT, et al. American Urological Association Guideline: Management of Benign Prostatic Hyperplasia 2010. [en línea]. Dirección URL: http://www.auanet.org/ benign-prostatic-hyperplasia-(2010-reviewed-and-validityconfirmed-2014).

2. Barry MJ, et al. The American Urological Association Symptom Index for Benign Prostatic Hyperplasia. DOI: https://doi. org/10.1016/j.juro.2016.10.071

3. Roehrborn CG. Pathology of benign prostatic hyperplasia. Int J Impot Res 2008;20(Suppl 3):S11-8.

4. Gallegos PJ, Frazee LA. Anticholinergic therapy for lower urinary tract symptoms associated with benign prostatic hyperplasia. DOI: https://doi.org/10.1592/phco.28.3.356

5. Chung DE, Kaplan SA. Current role for combination therapy in male LUTS. Arch Esp Urol 2010;63(5):323-32.

6. Novara G, et al. Individual patient data from registrational trials of silodosin in the treatment of non-neurogenic male lower urinary tract symptoms (LUTS) associated with benign prostatic hyperplasia (BPH): subgroup analyses of efficacy and safety data. DOI: https://doi.org/10.1111/bju.12906

7. Seki $\mathrm{N}$, et al. Non-inferiority of silodosin $4 \mathrm{mg}$ once daily to twice daily for storage symptoms score evaluated by the International Prostate Symptom Score in Japanese patients with benign prostatic hyperplasia: a multicenter, randomized, parallel-group study. Int J Urol. DOI: https:// doi.org/10.1111/iju.12680

8. Choo $M$, et al. Safety and efficacy of 8-mg once-daily vs 4-mg twice-daily silodosin in patients with lower urinary tract symptoms suggestive of benign prostatic hyperplasia (SILVER Study): a 12-week, double-blind, randomized, parallel, multicenter study. DOI: https://doi.org/10.1016/j. urology.2013.11.013

9. Shirakawa T, et al. Silodosin versus naftopidil in Japanese patients with lower urinary tract symptoms associated with benign prostatic hyperplasia: a randomized multicenter studyDOI: https://doi.org/10.1111/iju.12055

10. Yamaguchi $\mathrm{K}$, et al. Silodosin versus naftopidil for the treatment of benign prostatic hyperplasia: a multicenter randomized trial. DOI: https://doi.org/10.1111/iju.12160

11. Pande $S$, et al. Evaluation of silodosin in comparison to tamsulosin in benign prostatic hyperplasia: a randomized controlled trial. Indian J Pharmacol 2014;46(6):601-7.

12. Keten $\mathrm{T}$, et al. Determination of the efficiency of $8 \mathrm{mg}$ doxazosin $\mathrm{XL}$ treatment in patients with an inadequate response to $4 \mathrm{mg}$ doxazosin $\mathrm{XL}$ treatment for benign prostatic hyperplasia. DOI

13. Manjunatha $\mathrm{R}$, et al. A randomized, comparative, openlabel study of efficacy and tolerability of alfuzosin, tamsulosin and silodosin in benign prostatic hyperplasia. Indian J Pharmacol. 2016;48(2):134-40.

14. Kumar S, Kondareddy C, Ganesamoni R, Nanjappa B, Singh $S$. Randomized controlled trial to assess the efficacy of the combination therapy of alfuzosin and tadalafil in patients with lower urinary tract symptoms due to benign prostatic hyperplasia. DOI: https://doi.org/10.1111/luts.12016

15. Masumori N, et al. a-1-blocker tamsulosin as initial treatment for patients with benign prostatic hyperplasia: 5-year outcome analysis of a prospective multicenter study. DOI: https://doi.org/10.1111/j.1442-2042.2012.03165.x

16. Kim JJ, et al. Efficacy and tolerability of tamsulosin 0.4 $\mathrm{mg}$ in Asian patients with lower urinary tract symptoms secondary to benign prostatic hyperplasia refractory to tamsulosin $0.2 \mathrm{mg}$ : a randomized placebo controlled trial. DOI: https://doi.org/10.1111/iju.12412

17. Takeshita $\mathrm{H}$, et al. Randomized crossover comparison of the short-term efficacy and safety of single half-dose silodosin and tamsulosin hydrochoride in men with lower urinary tract symptoms secondary to benign prostatic hyperplasia. DOI: https://doi.org/10.1111/luts.12106

18. Karami $\mathrm{H}$, et al. Comparing monotherapy with tadalafil or tamsulosin and their combination therapy in men with benign prostatic hyperplasia: a randomized clinical trial. Urol J 2016;13(6):2920-6

19. Oelke $\mathrm{M}$, et al. Monotherapy with tadalafil or tamsulosin similarly improved lower urinary tract symptoms suggestive of benign prostatic hyperplasia in an international, randomised, parallel, placebo-controlled clinical trial. DOI: https://doi.org/10.1016/j.eururo.2012.01.013

20. Perumal $\mathrm{C}$, et al. A comparison of the efficacy of naftopidil and tamsulosin hydrochloride in medical treatment of benign prostatic enlargement. Urol Ann 2015;7(1):74-78.

21. Gacci $M$, et al. A randomized, placebo-controlled study to assess safety and efficacy of vardenafil $10 \mathrm{mg}$ and tamsu- 
losin $0.4 \mathrm{mg}$ vs. tamsulosin $0.4 \mathrm{mg}$ alone in the treatment of lower urinary tract symptoms secondary to benign prostatic hyperplasia. DOI: https://doi.org/10.1111/j.17436109.2012.02718.x

22. Dmochowski $R$, et al. Urodynamic effects of once daily tadalafil in men with lower urinary tract symptoms secondary to clinical benign prostatic hyperplasia: a randomized, placebo controlled 12-week clinical trial. J DOI: https://doi. org/10.1016/j.juro.2012.11.025

23. Brock $\mathrm{G}$, et al. Tadalafil once daily in the treatment of lower urinary tract symptoms (LUTS) suggestive of benign prostatic hyperplasia (BPH) in men without erectile dysfunction. DOI: https://doi.org/10.1111/bju.12251

24. Takeda $\mathrm{M}$, et al. Tadalafil $5 \mathrm{mg}$ once-daily therapy for men with lower urinary tract symptoms suggestive of benign prostatic hyperplasia: results from a randomized, doubleblind, placebo-controlled trial carried out in Japan and Korea. https://doi.org/10.1111/iju.12410

25. Takeda M, et al. Tadalafil for the Treatment of Lower Urinary Tract Symptoms in Japanese Men with Benign Prostatic Hyperplasia: Results from a 12-week Placebo-controlled Dose-finding Study with a 42-week Open-label Extension. https://doi.org/10.1111/j.1757-5672.2012.00144.x

26. Yokoyama $\mathrm{O}$, et al. Tadalafil once daily for lower urinary tract symptoms suggestive of benign prostatic hyperplasia: a randomized placebo- and tamsulosin-controlled 12-week study in Asian men. https://doi.org/10.1111/j.14422042.2012.03130.x

27. Singh DV, et al. A comparative randomized prospective study to evaluate efficacy and safety of combination of tamsulosin and tadalafil vs. tamsulosin or tadalafil alone in patients with lower urinary tract symptoms due to benign prostatic hyperplasia. DOI: https://doi.org/10.1111/ jsm.12357

28. Regadas RP, et al. Urodynamic effects of the combination of tamsulosin and daily tadalafil in men with lower urinary tract symptoms secondary to benign prostatic hyperplasia: a randomized, placebo-controlled clinical trial. Int Urol Nephrol 2013;45(1):39-43.

29. Casabe A, et al. Efficacy and safety of the coadministration of tadalafil once daily with finasteride for 6 months in men with lower urinary tract symptoms and prostatic enlargement secondary to benign prostatic hyperplasia. DOI: https://doi.org/10.1016/j.juro.2013.09.059

30. Fawzi A, et al. Sildenafil citrate in combination with tamsulosin versus tamsulosin monotherapy for management of male lower urinary tract symptoms due to benign prostatic hyperplasia: A randomised, double-blind, placebo-controIled trial. DOI: https://doi.org/10.1016/j.aju.2016.11.001

31. Joo KJ, et al. Comparison of [alpha]-blocker monotherapy and [alpha]-blocker plus 5[alpha]-reductase inhibitor combination therapy based on prostate volume for treatment of benign prostatic hyperplasia. DOI: https:// doi.org/10.1177/147323001204000308
32. Roehrborn C, et al. Influence of baseline variables on changes in International Prostate Symptom Score after combined therapy with dutasteride plus tamsulosin or either monotherapy in patients with benign prostatic hyperplasia and lower urinary tract symptoms: 4-year results of the CombAT study. DOI: https://doi.org/10.1111/bju.12500

33. Odusanya BO, et al. Short-term Effect of Tamsulosin and Finasteride Monotherapy and their Combination on Nigerian Men with Benign Prostatic Hyperplasia. Niger J Surg 2017;23(1):5-10.

34. Konstantinidis $C$, et al. Lower urinary tract symptoms associated with benign prostatic hyperplasia: combined treatment with fesoterodine fumarate extended-release and tamsulosin--a prospective study. https://doi.org/10.1159/000345050

35. Cai J-L, et al. Efficacy and safety of medium-to-long-term use of tolterodine extended release with or without tamsulosin in patients with benign prostate hyperplasia and larger prostate size: a double-blind, placebo-controlled, randomized clinical trial. Chin Med J 2016;129(24):2899-2906.

36. Ryu $Y$, et al. Comparison of tamsulosin plus serenoa repens with tamsulosin in the treatment of benign prostatic hyperplasia in Korean men: 1-year randomized open label study. DOI: https://doi.org/10.1159/000366521

37. Argirovic A, Argirovic D. Does the addition of Serenoa repens to tamsulosin improve its therapeutical efficacy in benign prostatic hyperplasia? Vojnosanit Pregl 2013;70(12):1091-6.

38. Morgia G, et al. Serenoa repens, lycopene and selenium versus tamsulosin for the treatment of LUTS/BPH. An Italian multicenter double-blinded randomized study between single or combination therapy (PROCOMB trial). DOI: https://doi. org/10.1002/pros.22866

39. Song CS, et al. Effect of Longbishu capsule () plus doxazosin on benign prostatic hyperplasia: a randomized controlled trial. Chin J Integr Med 2014;20(11):818-22.

40. Barrera M. Diferencias estadísticamente significativas vs. relevancia clínica. Rev CES Med 2008;22(1):89-96.

41. Abrams P, et al. Evaluation and Treatment of Lower Urinary Tract Symptoms in Older Men. DOI: DOI: https://doi. org/10.1016/j.juro.2012.11.021

42. Barragán-Arteaga I, Reyes-Vallejo L. Combination therapy for the treatment of lower urinary tract symptoms in men. Rev Mex Urol 2016;76(6):360-9.

43. NICE Clinical Guidelines. National Clinical Guideline Centre. The Management of Lower Urinary Tract Symptoms in Men. London: Royal College of Physicians (UK); 2015. [en línea]. Dirección URL: https://www.ncbi.nlm.nih.gov/books/ NBK65073/.

44. Yeo JK, et al. Korean clinical practice guideline for benign prostatic hyperpla. DOI: https://doi.org/10.4111/icu.2016.57.1.30

45. Singapore Urological Association Male Lower Urinary Tract Symptoms/Benign Prostatic Hyperplasia Guidelines Committee 2015. Singapore Urological Association Clinical Guidelines for Male Lower Urinary Tract Symptoms/Benign Prostatic Hyperplasia. Singapore Med J. doi: 10.11622/smedj.2017082 
46. Nunes RV, et al. Treatment of benign prostatic hyperplasia. Rev Assoc Med Bras. DOI: http://dx.doi.org/10.1590/18069282.63.02.95

47. López-Ramos H, et al. Guía de manejo de la hiperplasia prostática benigna. Sociedad Colombiana de Urología 2014. DOI: 10.1016/j.uroco.2015.04.005

48. Consejo de Salubridad General. Guía de Práctica Clínica. Diagnóstico y tratamiento de la hiperplasia prostática benigna [en línea]. Dirección URL: http://www.cenetec.salud.gob. $\mathrm{mx} /$ descargas/gpc/CatalogoMaestro/176_GPC_HIPERPLASIA_PROSTATICA/Gpc_hipertrofia_prostatica.pdf. [Consulta: 5 de julio 2108].

49. Dahm P, et al. Comparative effectiveness of newer medications for lower urinary tract symptoms attributed to benign prostatic hyperplasia: a systematic review and meta-analysis. DOI: https://doi.org/10.1016/j.eururo.2016.09.032

\section{AVISO IMPORTANTE}

La Revista Mexicana de Urología se convierte en una publicación solo digital, con todas las ventajas que los medios y dispositivos electrónicos ofrecen. Usted podrá revisar la información mediante el sitio web (www.revistamexicanadeurologia.org.mx) o descargando la app para Android o iPhone.

Para consultar el texto completo de los artículos deberá registrarse por una sola vez con su correo electrónico, crear una contraseña, indicar su nombre, apellidos y especialidad.

Esta información es indispensable para saber qué consulta y cuáles son sus intereses, y poder en el futuro inmediato satisfacer sus necesidades de información. 